\title{
Direct Detection of Kaluza-Klein Particles in Neutrino Telescopes
}

\author{
Ivone F. M. Albuquerque, ${ }^{1}$ Gustavo Burdman, ${ }^{1}$ Christopher A. Krenke, ${ }^{2,3}$ and Baran Nosratpour ${ }^{2}$ \\ ${ }^{1}$ Instituto de Física, Universidade de São Paulo, São Paulo, Brazil \\ ${ }^{2}$ Department of Physics, University of Arizona, Tucson, AZ 85721, USA \\ ${ }^{3}$ Department of Physics, University of Maryland, College Park, MD 20742, USA
}

\begin{abstract}
In theories with universal extra dimensions (UEDs), all standard model fields propagate in the bulk and the lightest state of the first Kaluza-Klein (KK) level can be made stable by imposing a $Z_{2}$ parity. We consider a framework where the lightest KK particle (LKP) is a neutral, extremely weakly interacting particle such as the first KK excitation of the graviton, while the next-to-lightest KK particle (NLKP) is the first KK mode of a charged right-handed lepton. In such a scenario, due to its very small couplings to the LKP, the NLKP is long-lived. We investigate the production of these particles from the interaction of high energy neutrinos with nucleons in the Earth, and determine the rate of NLKP events in neutrino telescopes. Using the Waxman-Bahcall limit for the neutrino flux, we find that the rate can be as large as a few hundreds events a year for realistic values of the NLKP mass.
\end{abstract}

PACS numbers: 11.30.pb, 13.15+g, 12.60.jv, 95.30.Cq

\section{INTRODUCTION}

Although the standard model (SM) is a successful description of the energy scales experimentally probed so far, it is expected that new physics will appear at the $\mathrm{TeV}$ scale. This is precisely the energy regime soon to be studied at the Large Hadron Collider (LHC). It is also the natural scale for the dynamical origin of electroweak symmetry breaking, as well as for the solution of the hierarchy problem. Typical solutions of these problems involve either symmetries (e.g. supersymmetry), some dynamical mechanism of electroweak symmetry breaking (e.g. technicolor), or a combination of symmetry and dynamics (e.g. little Higgs). In a somewhat different class are extensions of the SM involving compact extra dimensions. In Large Extra Dimensions [1] only gravity propagates in the extra dimensional bulk, and the true fundamental scale of gravitation is $O(1) \mathrm{TeV}$. On the other hand, in theories with one curved extra dimension [3], gravity is weak at the $\mathrm{TeV}$ scale due to the warping produced by the bulk metric.

Here we consider a more generic brand of extra dimensional theories, universal extra dimensions (UEDs), where all fields propagate in the extra dimensional bulk [2]. Its main motivation is phenomenological: if compact extra dimensions exist and all fields propagate in them, the inverse compactification radius could be just above the weak scale, setting the stage for a lot of new physics possibilities at the $\mathrm{TeV}$ scale. Furthermore, adding a mild assumption, the presence of a reflection symmetry leading to a $Z_{2}$-parity, UED theories are endowed with a candidate for dark matter: the lightest KK particle or LKP.

Although at leading order the spectrum of each KK level is degenerate, it splits under radiative corrections, as well as when generic higher dimensional operators are taken into account [4]. In theories with one extra di- mension, if only the loop contributions coming from the physics below the cutoff are considered, one obtains the spectrum of the minimal UED standard model (mUED) of Ref. [4]. In this case, the LKP is most likely to be the first KK mode of the photon $\gamma^{(1)}$. Other possibilities for the LKP include the first KK mode of the graviton $G^{(1)}$ [5], and (in theories where neutrino masses are Dirac) the first KK excitation of the right-handed neutrino $\tilde{N}^{(1)}$ [6]. Other light particles include the KK excitation of a right-handed charged lepton, $\ell^{(1)}$, and the charged Higgs KK mode [7]. The splitting between the LKP and $\ell^{(1)}$ is typically only a few $\mathrm{GeV}$, depending on the choice of parameters [26].

The mUED spectrum is merely illustrative, and ultraviolet physics contributions to boundary terms could significantly alter it, making for instance, $\ell^{(1)}$ the NLKP, while either $G^{(1)}$ or $\tilde{N}^{(1)}$ remains the LKP. In such a scenario, the decay of the NLKP to the LKP would be highly suppressed, making the NLKP lifetime very large. We will consider this possibility in this paper. This situation is analogous to what happens in some supersymmetric scenarios (e.g. gauge mediation) where the gravitino is the lightest supersymmetric particle and a right handed charged slepton is the next to lightest one. The phenomenology associated to a long-lived $\ell^{(1)}$ includes highly ionizing tracks at colliders. It also implies that $\ell^{(1)}$ can be produced by the interactions of high energy neutrinos with the earth and propagate through it until reaching a detector, in very close analogy to the case of NLSP sleptons studied in Refs. [9, 10, 11, 12, 13].

We will show that interactions of high energy neutri$\operatorname{nos}\left(\mathrm{E}_{\nu}>10^{5} \mathrm{GeV}\right)$ with nucleons in the Earth will produce pairs of NLKPs. The rate of events will allow the discovery of the latter in $\mathrm{km}^{3}$ neutrino telescopes. This analysis follows the same steps as for NLSP detection [9, 12]. The crucial observation is the same as for the NLSP, the small NLKP production cross section is com- 
pensated by its large range. The NLKP loses much less energy while traveling through the Earth when compared to SM leptons. This allows the detection of NLKPs that are produced far away from the detector.

As the NLKPs are produced in pairs, the main background consists of di-muon events. We will show that there are at least two ways to separate these from the signal. For lower mass NLKPs, the measured energy spectrum will have a bump in the region from $10^{3}$ to $10^{4} \mathrm{GeV}$ due to the fact that the energy loss in the detector will resemble the one from lower energy muons. In addition, for both low or high mass NLKPs, the separation between the pair that crosses the detector will be larger than the one for the di-muon pair, and will allow to distinguish the signal from the background.

This paper is organized as follows: we first determine the NLKP production cross section; in Section III we describe the NLKP energy loss while traveling through the Earth; the analysis of the signal and comparison with the background are discussed in Section IV] and the conclusions follow in the last section.

\section{NLKP PRODUCTION}

In this section, we compute the production cross section for the NLKP pair. Due to the presence of the $Z_{2^{-}}$ parity, all KK modes produced will eventually cascade down to a NLKP. Since KK modes are produced in pairs, KK production initiated by $\nu N$ scattering will result in a pair of NLKPs. The dominant process for $\nu-N$-initiated KK production involves the t-channel production of a left handed lepton KK mode $L_{i}^{(1)}$ (with generation index $i$ ) and a quark KK mode $\left(Q^{(1)}\right)$ via a gauge boson KK mode $\left(W^{(1)}\right)$. This process is analogous to the charged current (CC) in the SM. We also include the subdominant process which is analogous to the neutral current process in the SM. This involves the exchange of a neutral gauge boson KK mode $\left(Z^{(1)}\right)$. These processes are shown in Figure 1

The neutrino, which is always left-handed, can interact with a left handed down-type quark (a) or with a right-handed up-type antiquark (b). This results in the partonic cross sections:

$$
\begin{aligned}
\frac{d \sigma^{(\mathrm{a})}}{d t}= & \frac{8 \pi \alpha^{2}}{\sin ^{4} \theta_{W}} \frac{\left(s-m_{L_{i}^{(1)}}^{2}-m_{Q^{(1)}}^{2}\right)}{s^{2}\left(t^{2}-M_{W^{(1)}}^{2}\right)^{2}} \\
\frac{d \sigma^{(\mathrm{b})}}{d t}= & \frac{8 \pi \alpha^{2}}{\sin ^{4} \theta_{W}} \times \\
& \frac{\left[m_{L_{i}^{(1)}}^{2} m_{Q^{(1)}}^{2}+u^{2}-u\left(m_{L_{i}^{(1)}}^{2}+m_{Q^{(1)}}^{2}\right)\right]}{s^{2}\left(t^{2}-M_{W^{(1)}}^{2}\right)^{2}}
\end{aligned}
$$

where $s, t$ and $u$ are the usual Mandelstam variables, and $m_{L_{i}^{(1)}}, m_{Q^{(1)}}$ and $M_{W^{(1)}}$ are the $L_{i}^{(1)}, Q^{(1)}$ and $W^{(1)}$
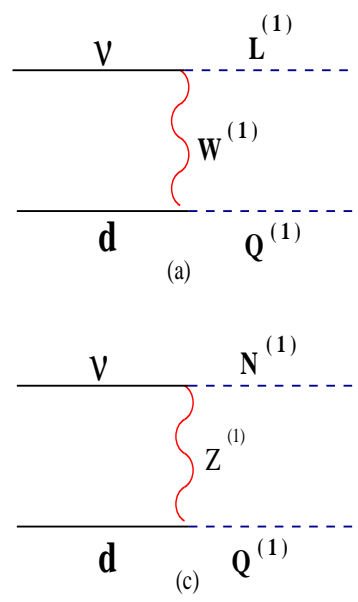

FIG. 1: Feynman diagrams for KK mode production in $\nu N$ collisions. Charged current (charged gauge boson KK mode) interactions: (a) Left-left interaction requiring the insertion of the gauge KK mode mass in the t-channel line. (b) Left-right interaction. Neutral current: (c), (d). There are analogous diagrams for anti-neutrinos as well as for strange and charm initial quarks.

masses, repectively. The subdominant neutral gauge boson KK mode $\left(Z^{(1)}\right)$ exchange is shown in Figure 1 (c)(d). Each of these processes will produce a $L_{i}^{(1)}$ and a $Q^{(1)}$ and both of these particles will promptly produce a decay chain ending with a $\ell_{i}^{(1)}$.

Bounds from direct searches from the Tevatron, as well as from electroweak precision constrains [2], result in $R^{-1}>300 \mathrm{GeV}$ for $5 \mathrm{D}$, while for $6 \mathrm{D}$ is $R^{-1}>500 \mathrm{GeV}$. We will assume three illustrative values for the NLKP mass: 300,600 and $900 \mathrm{GeV}$. Finally, we need to specify the cutoff of the theory. Using naive dimensional analysis, we find for the $5 \mathrm{D}$ case, that $\Lambda R \sim 8 \pi / g^{2}$, where $g$ is the strong coupling constant. We then take $\Lambda R \simeq 20$ as a sharp cutoff, i.e. we include no contributions from energies above $20 / R$. In order to evaluate the uncertainty introduced by this procedure in the cross section calculation, we scanned values of $\Lambda R$ up to 30 , with no significant effects in the results.

The NLKP production cross section is shown in Figure 2 as a function of the neutrino energy. For comparison, the SM charged current (top gray curve) and the di-muon (solid red curve) background cross sections are also shown. As expected, the NLKP production cross sections $\left(\sigma_{\mathrm{NLKP}}\right)$ are significantly lower than the SM one. However, depending on the neutrino energy and the $L_{i}^{(1)}$ mass, it can be larger than the di-muon background. In the next section we will show that the the fact that $\sigma_{\mathrm{NLKP}}$ is rather suppressed (as compared to the SM one) will be compensated by the sizable NLKP range resulting from the combination of its long lifetime and small energy loss.

It is also interesting to compare the $\sigma_{\mathrm{NLKP}}$ to the NLSP production as obtained in Ref. [9]. The NLKP production is significantly larger than the one for NLSPs, which 


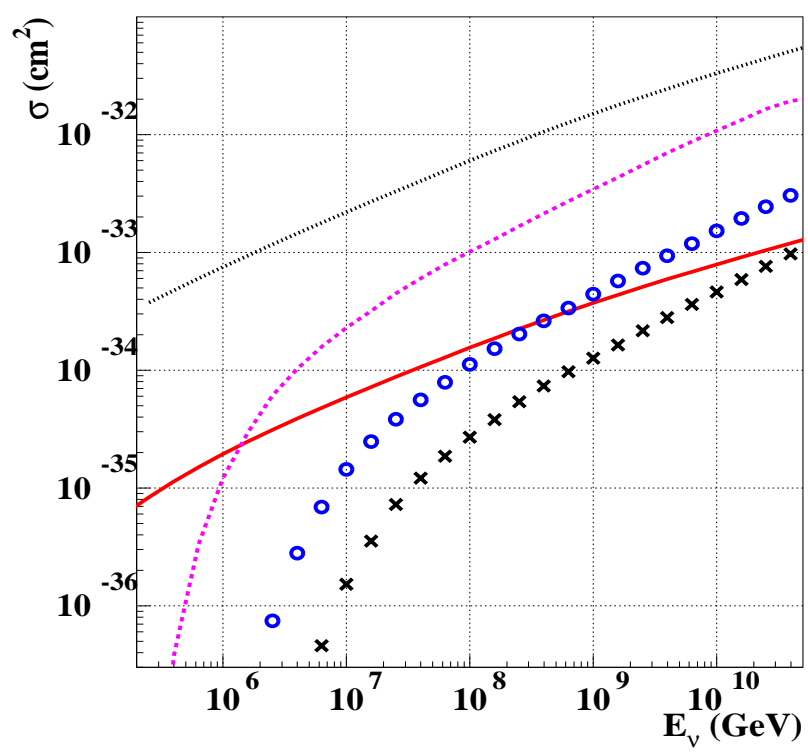

FIG. 2: $\nu N$ cross sections vs. the energy of the incident neutrino. The violet dashed, blue circled and black crossed lines correspond respectively to 300, 600 and $900 \mathrm{GeV}$ NLKPs. The top gray curve corresponds to the SM charged current interactions and the full red one to the di-muon background.

translates into a larger number of events at the detector, as we will see below.

\section{NLKP ENERGY LOSS}

After production, the NLKPs lose energy due to ionization and radiation processes. The average energy loss is given by [14]:

$$
-\frac{d E}{d x}=a(E)+b(E) E
$$

where $a(E)$ represents the ionization losses, and $b(E)$ the contributions from different radiation processes. The latter includes bremsstrahlung, pair production and photonuclear interactions. There is also energy loss due to weak interactions, but this will only be of importance at very high energy [18], and we will neglect it for the remaining of this work.

At the high energies where NLKPs can be produced, radiation losses dominate over ionization. Among radiation processes, both pair production and bremsstrahlung become less important for heavy particles. Although photonuclear processes dominate tau lepton propagation losses [15, 16], a mass suppression will occur for leptons of much heavier masses [12, 17].
In order to determine the NLKP energy losses, we follow closely the calculations done for NLSP propagation in Refs. [12] and 17]. Radiation losses dominate above a propagating energy of $1 \mathrm{TeV}$. Among them, pair production and bremsstrahlung are less important for the NLKP when compared to photo-nuclear interactions, as can be seen in Figure 3. Even so, the energy loss due to photo-nuclear interactions is suppressed by the NLKP mass. As mentioned in Ref. 9] and shown explicitely in Refs. [12] and [17], the important energy region for this process is the one at low photon virtuality $Q^{2}$. The reason is that the structure function involved in the process is determined by a cross section which is dominated by physics at low $Q^{2} \simeq 1 \mathrm{GeV}^{2}$. However, due to the large NLKP mass, the minimum value for the photon virtuality will be larger, therefore avoiding the effects of resonances and other nonperturbative processes which occur at lower $Q^{2}$. This is in contrast to the case of the $\tau$ lepton, where the resonant region still dominates and results in a much larger photo-nuclear energy loss.

Figure 3 shows the radiation loss term of eq. (3.4) versus neutrino energy for muons, taus and the $300 \mathrm{GeV}$ NLKP. As expected, the photo-nuclear process dominates the NLKP radiation loss. However, it is still quite suppressed due to the NLKP heavy mass and the total energy loss is still considerably below the one for SM leptons. Energy suppression will be enhanced for heavier NLKP mass.

We then conclude that the NLKP energy loss is quite suppressed in comparison with SM leptons. As we will see below, this means that its range through the Earth is much larger, allowing for the detection of NLKPs that have been produced hundreds or even thousands of kilometers from the detector.

\section{NLKP SIGNALS AND RATE IN NEUTRINO TELESCOPES}

\section{A. Neutrino Flux}

The NLKP event rate in neutrino telescopes depends on the incoming neutrino flux. This is largely determined by the high energy cosmic ray spectrum [19]. There are other potentially relevant sources of the neutrino flux, such as atmospheric charm production [20]. For the purpose of this work we will neglect these other contributions, only considering the flux of cosmic neutrinos, for which we use two alternative estimates: the work of Waxman and Bahcall (WB) 21 and the one of Manheim, Proterhoe and Rachen (MPR) 22]. The integrated number of events resulting from the MPR limit is considerably larger than the WB. We find our NLKP rates assuming each of these limits as our incoming neutrino flux. All plots are produced assuming the WB limit as our neutrino flux.

Waxman and Bahcall fix the cosmic ray spectrum to a power law curve with spectral index -2 . The neutrino 

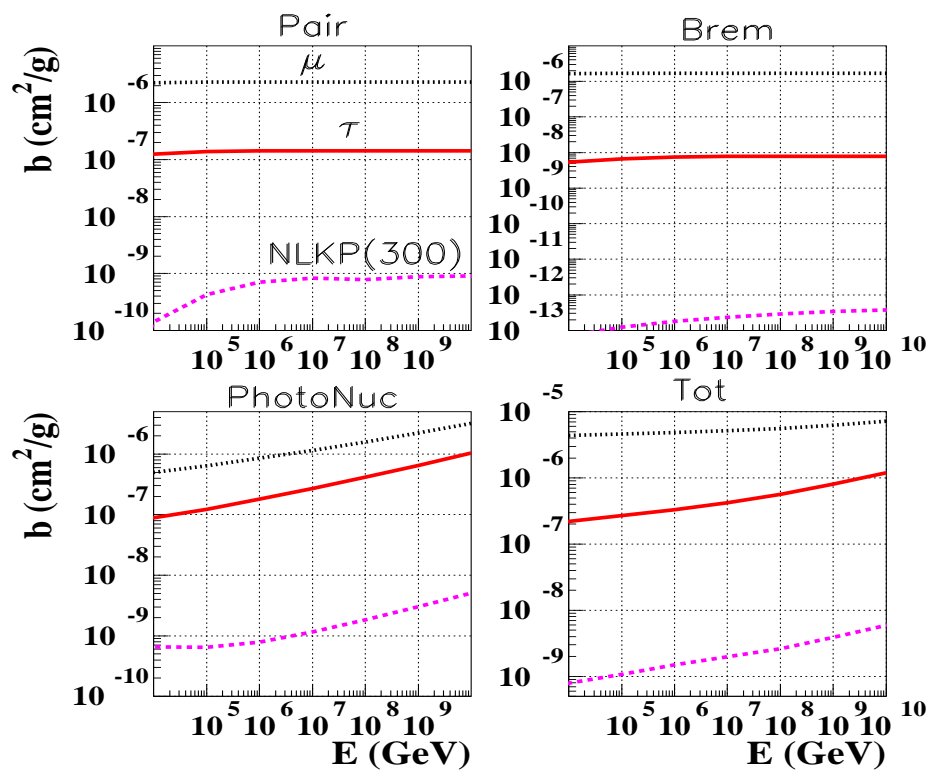

FIG. 3: Radiation energy loss $\mathrm{b}(\mathrm{E})$ parameter due to pair production, bremsstrahlung and photonuclear processes for muon, tau and a $300 \mathrm{GeV}$ NLKP. The plot labeled "Tot" is the sum of all contributions. The muon, tau and NLKP curves are as labeled in the first plot. Heavier NLKPs will have lower $\mathrm{b}(\mathrm{E})$ parameters.

upper limit is deduced assuming that each nucleon will interact with photons and produce a pion. The charged pions will then decay producing neutrinos. Their argument requires that the sources are optically thin, which means that most of the protons escape the source without interacting. As a result, the neutrino upper limit is given by

$$
\left(\frac{d \phi_{\nu}}{d E}\right)_{\mathrm{WB}}=\frac{(1-4) \times 10^{-8}}{E^{2}} \mathrm{GeV} \mathrm{cm}^{-2} \mathrm{~s}^{-1} \mathrm{sr}-1,
$$

where the range in the coefficient depends on the cosmological evolution of the sources. The evolution accounts for the source activity and redshift energy loss due to the cosmological expansion. We take the upper end as the neutrino flux incoming through the Earth.

On the other hand, instead of taking a fixed power law behaviour for all cosmic ray spectrum, Manheim, Proterhoe and Rachen determine the spectrum at each energy directly from data. Here we consider the limit MPR obtain assuming optically thin sources, although they also determine a limit for optically thick sources (See comments about optically thick sources in [19]). Figure 4 shows both WB and MPR limits for the muon plus antimuon neutrino flux.

As seen in Section II the NLKP production is inde-

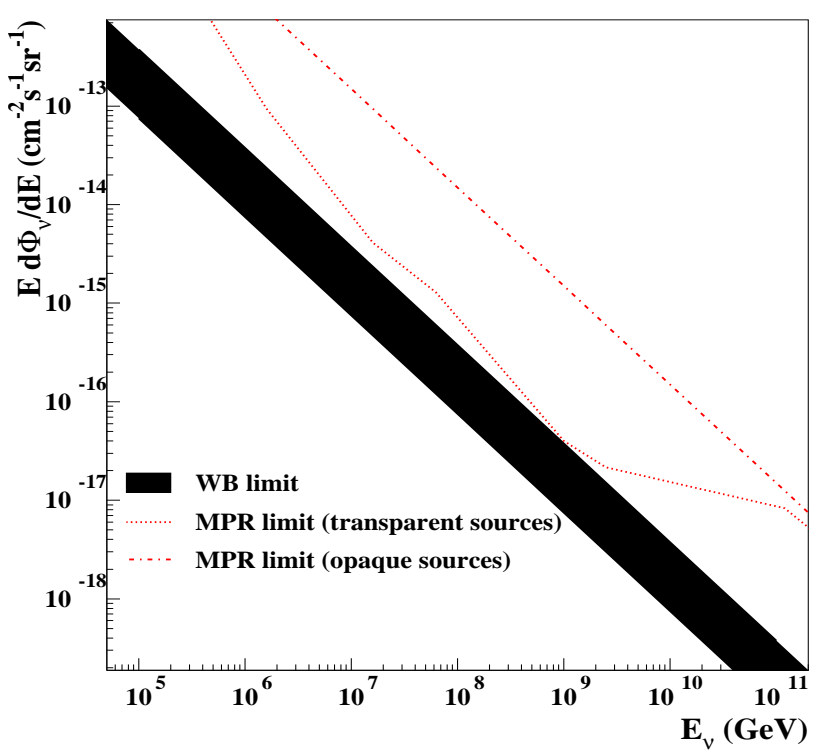

FIG. 4: Upper bound on differential neutrino flux as calculated by WB [21] (shaded area) and MPR [22] (red lines). The WB limit ranges from the limit with no cosmological evolution (upper edge) and with cosmological evolution (lower edge). The MPR limit is shown for optically thin sources (red dotted line) as used in this paper and for optically thick sources (red dot-dashed line).

pendent of the initial neutrino flavor. For this reason we consider both electron and muon neutrinos, and neutrino mixing does not affect our results.

\section{B. NLKP Signals}

We now have all the ingredients to determine the NLKP rate at neutrino telescopes. In order to understand the signal in detail, we performed a Monte Carlo simulation generating approximately 30,000 events for each NLKP mass $(300,600$ and $900 \mathrm{GeV})$.

Once the incoming neutrino flux is determined, an interaction point is randomly chosen based on the NLKP production probability. This results from a convolution of the neutrino survival probability with the probability of interacting and producing a NLKP. The neutrino survival probability $P_{S}$ is given by $\exp \left(\int n d l\right)$, where $n$ is the Earth number density and $l$ is the distance the neutrino travels. We use the Earth density profile as described in [23, 24].

The primary particles $\left(L_{i}^{(1)}\right.$ and $Q^{(1)}$ produced in the neutrino interaction) angular distribution at the $\mathrm{CM}$ is randomly determined based on the differential produc- 


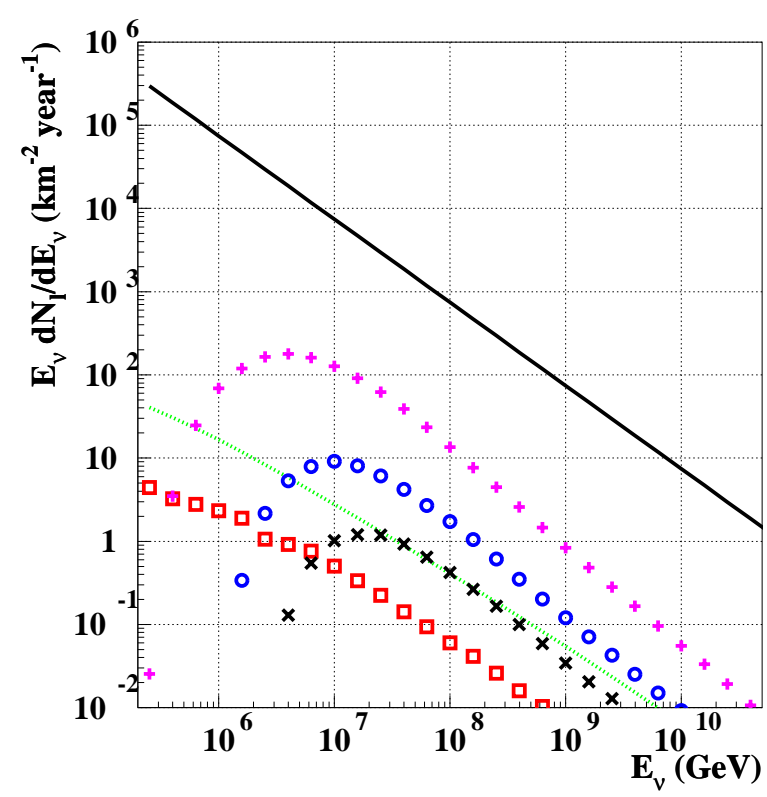

FIG. 5: NLKP pair event energy distribution per $\mathrm{km}^{2}$, per year, at the detector. Plus violet line corresponds to 300 $\mathrm{GeV}$; blue circled line to $600 \mathrm{GeV}$ and crossed black line to $900 \mathrm{GeV}$ NLKP. For reference the neutrino flux at earth (full black line); and the $\mu$ (dotted green line) and the di-muon (squared red line) flux through the detector are also shown. In all cases we make use of the WB limit for the neutrino flux.

tion cross section. The center of mass (CM) angular distribution of the two NLKPs produced is assumed to be the same as the one between the two primary particles. This is a good approximation [12] for events with energy well above the production threshold where most of the event rate comes from. The events close to the production threshold have a broader angular distribution. These would enhance the separation differences between signal and background and therefore make our results conservative. The $\mathrm{CM}$ angular distribution is then boosted to the laboratory frame.

Once the NLKPs are produced their propagation through the Earth is simulated. Their energy loss - which is mass dependent - is taken into account. The NLKP energy distribution as a function of neutrino energy is shown in Figure 5. As can be seen, the 300 and $600 \mathrm{GeV}$ NLKP event rate is much larger than the muon's for energies above NLKP production threshold. The $900 \mathrm{GeV}$ NLKP rate will be comparable with that for muons, but still larger than the di-muon background rate.

Although these are rather large rates, they do not directly translate into observed NLKPs due to the fact that NLKPs are hard to identify. Neutrino telescopes measure their energy in two ways 19, 25]: low energy events (be-
TABLE I: Number of events per $\mathrm{km}^{2}$ per year for different NLKP masses and neutrino fluxes at the Earth. The NLKP masses are 300, 600 and $900 \mathrm{GeV}$. The number of NLKP events are given for energies above threshold for production of a $L_{\ell}^{(1)}$ and a $Q^{(1)}$ while the muon rate for energies above $1000 \mathrm{GeV}$. The column $\mu^{+} \mu^{-}$corresponds to the di-muon background. No cuts were applied at this stage.

\begin{tabular}{l|ccccc}
\hline \hline & $\mu$ & $\mu^{+} \mu^{-}$ & & $L_{R}^{(1)} \bar{L}_{R}^{(1)}$ & \\
& & & $(300)$ & $(600)$ & $(900)$ \\
\hline WB & 552 & 30 & 489 & 21 & 3 \\
MPR & 39654 & 1914 & 1476 & 47 & 5 \\
\hline \hline
\end{tabular}

low $\sim 100 \mathrm{GeV}$ ) have their energy reconstructed from the track length, whereas for the more energetic ones the energy is reconstructed from the amount of Cerenkov light deposited in the photomultiplier tubes. Taking the Cerenkov radiation as proportional to the amount of deposited energy in the detector is a good approximation for SM leptons. But the NLKPs lose a lot less energy than SM leptons. Thus, if a NLKP track is assumed to be a SM lepton such as a muon, it will be assigned a much lower energy as such. For this reason and in order to compare event rates, the muon rate must be integrated from energies lower than the KK production threshold.

Table $\llbracket$ shows the event rate per year per $\mathrm{km}^{2}$ both for the WB flux, as well as for the MPR optically thin flux. The numbers are clearly encouraging for $\mathrm{km}^{3}$ neutrino telescopes. Two features will be important to distiguish the signal from the background : the separation between the pair of particles inside the detector; and - for lower mass NLKPs - a bump in the energy spectrum will appear. These features will be discussed at the end of this section.

\section{Di-muon Background}

Due to their large boost most NLKP pairs go through the detector in two well separated and approximately parallel tracks. Events well separated are produced far from the detector and as the production angle between them is small the tracks will be almost parallel. Therefore, single muons can be eliminated by a two track requirement. The main remaining background are dimuons. These are produced from charmed hadrons from the following chain :

$$
\nu N \rightarrow \mu^{-} H_{c} \rightarrow \mu^{-} \mu^{+} H_{x} \nu,
$$

where $H_{c}$ is a charm hadron produced from a muon neutrino CC interaction and $H_{x}$ can be either a strange or non-strange hadron.

The cross section for charm production from a neutrino interaction was calculated in Ref. [12], as well as 
the di-muon energy loss, propagation and separation at the detector. In what follows we reproduce these results, and compare with the NLKP signal.

\section{NLKPs Separation}

The separation between the NLKPs will be given by the distance traveled times the angle $(\theta)$ between the pair in the laboratory frame. As the boost from CM to lab is large, $\theta$ is very small. However, this is compensated by the production being far away from the detector. The production point being typically a few $1000 \mathrm{~km}$ from the detector and $\theta \sim 10^{-4}-10^{-5}$ the separation between the two NLKPs will be a few tens to a few hundred meters.

On the other hand, di-muon events have to be produced close to the detector, otherwise they lose all their energy before arriving at it. For this reason their separation is typically smaller than the one for most of the signal events.

The separation distribution for each NLKP mass at the detector is shown in Figure 6. The simulated detector is placed at the same depth as the IceCube telescope [25]. We also show the di-muon background separation for comparison. While the dimuon separation is at most $\sim 100 \mathrm{~m}$, the pair of NLKP can be more than 100 meters apart. For instance, for a $300 \mathrm{GeV}$ NLKP, 52\% of the events are more than $50 \mathrm{~m}$ apart and $28 \%$ are more than $100 \mathrm{~m}$ apart. The di-muon background has only $8 \%$ with more than $50 \mathrm{~m}$ and $1.3 \%$ with more than $100 \mathrm{~m}$ separation. The 600 and $900 \mathrm{GeV}$ NLKPs have both around $60 \%$ of events with more than $50 \mathrm{~m}$ separation and around $42 \%$ with more than $100 \mathrm{~m}$ separation.

In order to estimate the statistical significance of the separation cut, we determine the $S / \sqrt{B}$ ratio, where $S$ and $B$ are respectively the number of signal and background events. We find that for the $300 \mathrm{GeV}$ NLKP, a requirement that the pair of NLKPs are at least 10 meters apart will yield a significance of 85 , ie, 436 of the 489 NLKPs will be more than 10 meters apart, while only 25 di-muons will have more than 10 meters separation. For the $600 \mathrm{GeV}$ NLKP, a requirement of $86 \mathrm{~m}$ separation will allow a $5 \sigma$ significance in one year, with 9 signal events and 3 di-muons. For the $900 \mathrm{GeV}$, the separation is harder, a $2 \sigma$ significance can be achieved in a year with a separation cut of $150 \mathrm{~m}$, while a $5 \sigma$ significance needs 5 years to be achieved.

\section{The NLKP Bump}

Another feature of the NLKP signal comes from the fact that these particles lose less energy than a SM lepton. This implies that NLKPs will have their energy reconstructed as if they where lower energy leptons. Figure 7 shows both NLKP and di-muon simulated energy distribution as they arrive at the detector. Although the NLKPs are more energetic than the di-muons, the
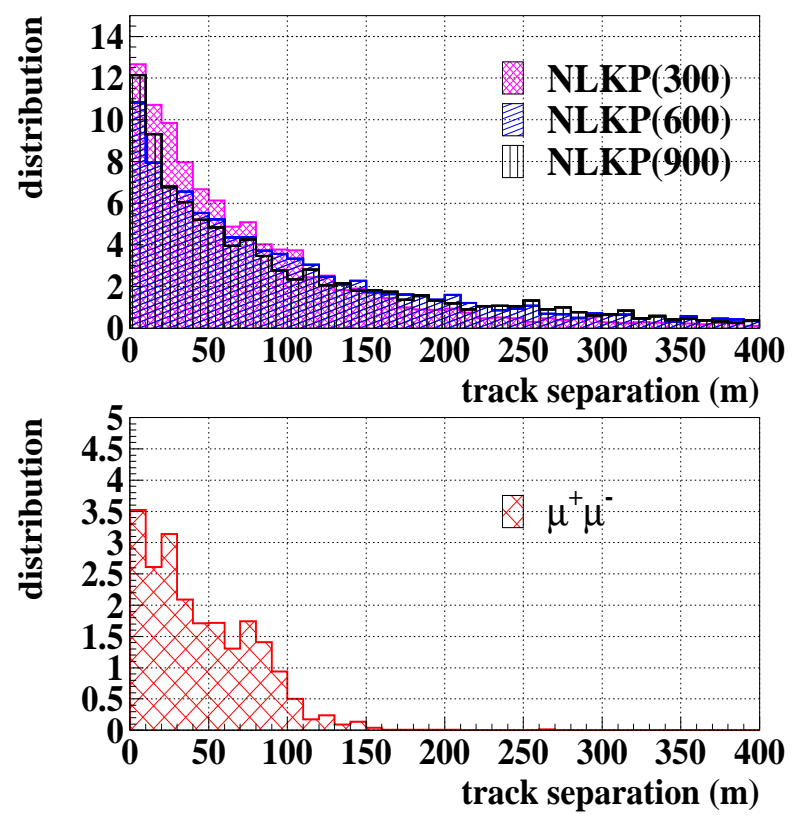

FIG. 6: Track separation distribution between NLKP pair (top) and for the di-muon background (bottom).

energy deposited in the PMTs will resemble lower energy muons and therefore they have to be compared with them. However this will generate a sizeable excess in the reconstructed energy spectrum, at least if the number of NLKP events is large enough. In order to understand how this feature will change the reconstructed energy spectrum, we simulate the reconstructed energy by taking all NLKPs as muons. This was done by determining the deposited energy in the detector and reconstructing this energy as if deposited by a muon. These events were then added to the SM muon energy spectrum. The consequence is that the high energy NLKPs will be reconstructed as lower energy events that will end up as a bump around energies of TeVs.

Figure 8 shows the energy distribution of the muon flux through the detector (top plot, blue circles) and the same distribution with the addition of $300 \mathrm{GeV}$ NLKPs reconstructed as muons. A visible "crown" with few events in each energy bin in the 1 to $100 \mathrm{TeV}$ region will clearly indicate the presence of KK particles. This feature will be enhanced when the NLKPs are included in the dimuon energy spectrum (bottom plot). When the signal is reconstructed as di-muons, a pronounced crown shows up in the reconstructed energy spectrum. This feature is observable for NLKPs in the lower mass range, since the rate of higher mass NLKPs would not be large enough to observably enhance the spectrum in the lower energy region. We expect this feature to be observable up to NLKP masses of about $\sim 600 \mathrm{GeV}$. Thus, for these lower mass NLKPs there will be two distinct ways to separate 


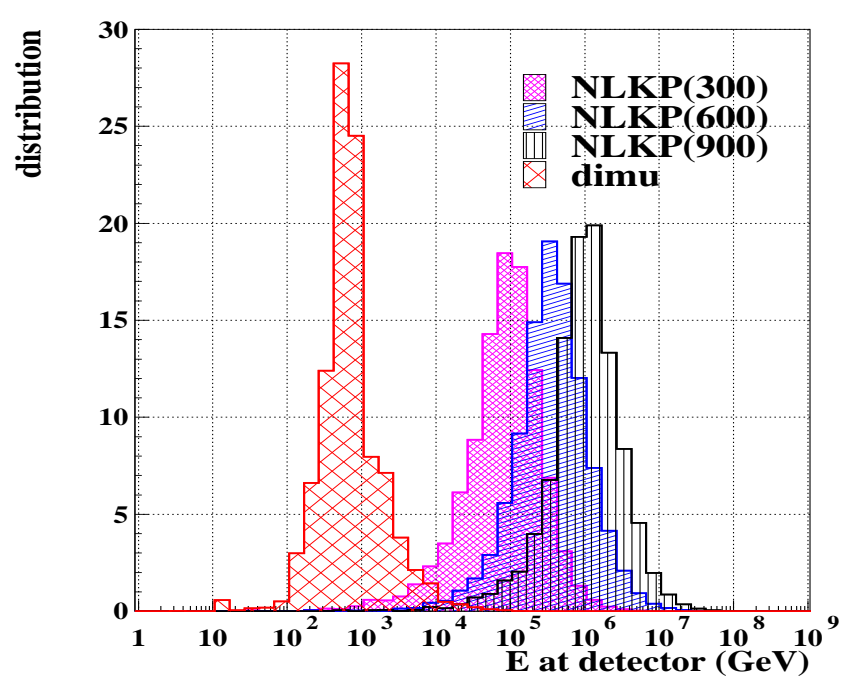

FIG. 7: Arrival energy distribution of the NLKP at the detector for $m_{L_{R}^{(1)}}=300,600$ and $900 \mathrm{GeV}$. Also shown is the arrival distribution for the di-muon background.

the signal from the main background.

\section{CONCLUSIONS}

We have shown that in a UED scenario where the NLKP is a the first KK mode of a right handed charged lepton, neutrino telescopes such as IceCube will be able to directly observe these $\ell_{i}^{(1)}$ 's up to masses of several hundred $\mathrm{GeV}$, perhaps even $1 \mathrm{TeV}$. This complements hadron collider searches, where signals for this UED scenario would consist of large missing energy, and perhaps one or two highly-ionizing tracks. The similarity of the UED signals with the analogous supersymmetric scenario, for instance with gravitino dark matter and a slepton NLSP, can make the identification of the underlying theory difficult. On the other hand, the event rate at neutrino telescopes coming from this UED scenario is considerably higher than the one resulting from the supersymmetric case and studied in Refs. [9, 12].
We have made a detailed study of the background and the signal, and shown that the track separation of NLKPs is a good discriminant with respect to the di-muon background. In addition, for the case of smaller NLKPs masses, we have shown that the NLKP signal results in a bump in the detected di-muon spectrum, since NLKPs lose energy similarly to lower energy muons. Combining this feature with the characteristic track separation of the signal tracks should enhance the statistical significance of a potential signal.
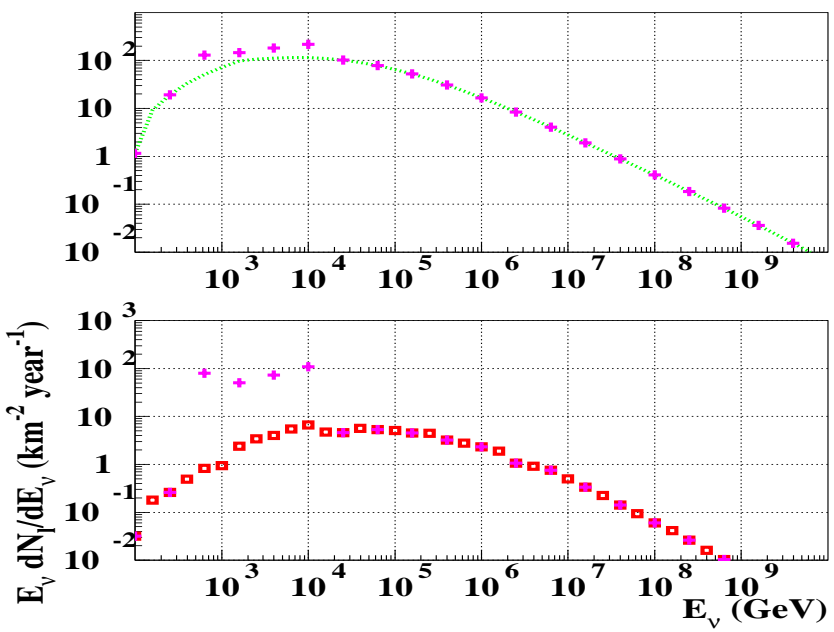

FIG. 8: Top: Energy distribution of the muon flux through the detector (blue circles) and the same flux plus the $300 \mathrm{GeV}$ NLKP spectrum reconstructed as muons (violet plus line). Bottom: Same as above but now using the di-muon flux through the detector.

Acknowledgments - The authors thank Z. Chacko for helpful discussions and for his collaboration at early stages of this work. I. A. and G. B. acknowledge the support of the State of São Paulo Research Foundation (FAPESP) and the Brazilian National Counsel for Technological and Scientific Development $(\mathrm{CNPq})$. C. K. and B. N. are supported by the NSF under grant PHY0408954 .
[1] N. Arkani-Hamed, S. Dimopoulos and G. R. Dvali, Phys. Lett. B 429, 263 (1998); N. Arkani-Hamed, S. Dimopoulos and G. R. Dvali, Phys. Rev. D 59, 086004 (1999).

[2] T. Appelquist, H-C. Cheng and B. A. Dobrescu, Phys. Rev. D 64, 035002 (2001).

[3] L. Randall and R. Sundrum, Phys. Rev. Lett. 83, 3370 (1999).
[4] H. C. Cheng, K. T. Matchev and M. Schmaltz, Phys. Rev. D 66, 036005 (2002) arXiv:hep-ph/0204342.

[5] J. L. Feng, A. Rajaraman and F. Takayama, Phys. Rev. Lett. 91, 011302 (2003) arXiv:hep-ph/0302215; J. L. Feng, A. Rajaraman and F. Takayama, Phys. Rev. D 68, 063504 (2003) arXiv:hep-ph/0306024; J. L. Feng, A. Rajaraman and F. Takayama, Phys. Rev. D 68, 
085018 (2003) arXiv:hep-ph/0307375.

[6] S. Matsumoto, J. Sato, M. Senami and M. Yamanaka, Phys. Lett. B 647, 466 (2007) arXiv:hep-ph/0607331; S. Matsumoto, J. Sato, M. Senami and M. Yamanaka, Phys. Rev. D 76, 043528 (2007) arXiv:0705.0934 [hep$\mathrm{ph}]$.

[7] J. A. R. Cembranos, J. L. Feng and L. E. Strigari, Phys. Rev. D 75, 036004 (2007) arXiv:hep-ph/0612157.

[8] G. Burdman, B. A. Dobrescu and E. Ponton, JHEP 0602, 033 (2006) arXiv:hep-ph/0506334; G. Burdman, B. A. Dobrescu and E. Ponton, Phys. Rev. D 74, 075008 (2006) arXiv:hep-ph/0601186.

[9] I. Albuquerque, G. Burdman and Z. Chacko, Phys. Rev. Lett. 92, 221802 (2004).

[10] X. J. Bi, J. X. Wang, C. Zhang and X. m. Zhang, Phys. Rev. D 70, 123512 (2004) arXiv:hep-ph/0404263.

[11] M. Ahlers, J. Kersten and A. Ringwald, JCAP 0607, 005 (2006) arXiv:hep-ph/0604188.

[12] I. Albuquerque, G. Burdman and Z. Chacko, Phys. Rev. D 75, 035006 (2007).

[13] M. Ahlers, J. I. Illana, M. Masip and D. Meloni, JCAP 0708, 008 (2007) arXiv:0705.3782 [hep-ph]]; M. H. Reno, I. Sarcevic and J. Uscinski, Phys. Rev. D 76, 125030 (2007) arXiv:0710.4954 [hep-ph]]; S. Ando, J. F. Beacom, S. Profumo and D. Rainwater, arXiv:0711.2908 [hep-ph].

[14] W. M. Yao et al. [Particle Data Group], J. Phys. G 33, 1 (2006).

[15] S. I. Dutta, M. H. Reno, I. Sarcevic and D. Seckel, Phys. Rev. D 63, 094020 (2001).

[16] E. V. Bugaev and Y. V. Shlepin, Phys. Rev. D 67, 034027
(2003)

[17] M. H. Reno, I. Sarcevic and S. Su, Astropart. Phys. 24, 107 (2005).

[18] Y. Huang, M. H. Reno, I. Sarcevic and J. Uscinski, Phys. Rev. D 74, 115009 (2006)

[19] For a detailed discussion of neutrino event rates see I. Albuquerque, J. Lamoureux and G. F. Smoot, Astrophys. J. Suppl. 141, 195 (2002).

[20] S. Ando, J. F. Beacom, S. Profumo and D. Rainwater, arXiv:0711.2908 [hep-ph].

[21] E. Waxman and J. N. Bahcall, Phys. Rev. D 59, 023002 (1999); J. N. Bahcall and E. Waxman, Phys. Rev. D 64, 023002 (2001).

[22] K. Mannheim, R. J. Protheroe and J. P. Rachen, Phys. Rev. D 63, 023003 (2001)

[23] R. Gandhi, C. Quigg, M. H. Reno and I. Sarcevic, Astropart. Phys. 5, 81 (1996); R. Gandhi, C. Quigg, M. H. Reno and I. Sarcevic, Phys. Rev. D 58, 093009 (1998).

[24] A. Dziewonski, in Encyclopedia of Solid Earth Geophysics, ed. J.D.E. Van Nostrand (New York: Reinhold), 331 (1989).

[25] J. Ahrens et al. [The IceCube Collaboration], Nucl. Phys. Proc. Suppl. 118, 388 (2003).

[26] In UED theories with two extra dimensions [8], the LKP is typically a neutral scalar adjoint, $B_{H}^{(1,0)}$. However, in principle the charged scalars $W_{H}^{(1,0) \pm}$ could be made lighter by higher dimensional operators, resulting in a similar situation as in 5D. We will not pursue this possibility here. 